\title{
EXPONENTIAL SPLITTING FOR UNBOUNDED OPERATORS
}

\author{
ESKIL HANSEN AND ALEXANDER OSTERMANN
}

\begin{abstract}
We present a convergence analysis for exponential splitting methods applied to linear evolution equations. Our main result states that the classical order of the splitting method is retained in a setting of unbounded operators, without requiring any additional order condition. This is achieved by basing the analysis on the abstract framework of (semi)groups. The convergence analysis also includes generalizations to splittings consisting of more than two operators, and to variable time steps. We conclude by illustrating that the abstract results are applicable in the context of the Schrödinger equation with an external magnetic field or with an unbounded potential.
\end{abstract}

\section{INTRODUCTION}

In this paper, we are concerned with approximating the solution of the linear evolution equation

$$
\dot{u}=L u=(A+B) u, \quad u(0)=u_{0},
$$

where $L, A$ and $B$ are unbounded operators. As a numerical method, we will employ an $s$-stage exponential splitting scheme, based on the linear operator

$$
S=\prod_{j=1}^{s} \mathrm{e}^{\gamma_{j} h A} \mathrm{e}^{\delta_{j} h B} .
$$

The numerical solution after one step of size $h \in \mathbb{R}$ is defined by $S u_{0}$, and it approximates the exact solution $u(h)=\mathrm{e}^{h L} u_{0}$ of the problem. The idea behind the splitting approach is that the action of the individual flows $\mathrm{e}^{h A}$ and $\mathrm{e}^{h B}$ on a vector can be computed more efficiently than the corresponding quantity $\mathrm{e}^{h L} u_{0}$. This is, for example, the case for the Schrödinger equation with an external magnetic field $b(x)=\nabla \times a(x)$ and a real potential $W(x)$.

The real coefficients $\gamma_{j}$ and $\delta_{j}$ in (1.2) are chosen in such a way that the method has classical order $p$. This is achieved by formally expanding $S$ and $\mathrm{e}^{h L}$ into Taylor series in $h$ and comparing the terms in the expansion up to order $p$. As long as the remainders of these Taylor expansions stay moderately bounded, this concept serves as a foundation of an error analysis. In the present study, we strive further and develop a rigorous framework that enables the derivation of optimal convergence orders even for unbounded operators.

Received by the editor February 29, 2008 and, in revised form, August 19, 2008.

2000 Mathematics Subject Classification. Primary 65M15, 65J10, 65L05, 35Q40.

Key words and phrases. Exponential splitting, splitting schemes, convergence, nonstiff order, unbounded operators, Schrödinger equation.

This work was supported by the Austrian Science Fund under grant M961-N13.

(C)2009 American Mathematical Society 
Splitting methods currently constitute a very active field. Recent surveys are given in the monograph [7] and the article [13]. There, convergence results are presented for the nonstiff situation with bounded operators. For unbounded operators, however, there still lacks a general convergence theory that yields optimal convergence orders. For a brief overview of the partial results connected to the special applications we refer to the following studies: Hyperbolic problems [2, 11, 16], parabolic problems 4, 8, 9, and Schrödinger equations [10, 12, 18.

The outline of our paper is as follows: In Section 2, we introduce the employed framework of $C_{0}$ groups and we present our main result. It states that, under standard regularity assumptions, the classical order of the splitting method is retained in the stiff case, without requiring any additional order condition. The crucial step for conducting the proof is to derive a suitable consistency for unbounded operators. This is achieved in Section 3. Generalizations to splittings involving more than two operators, to variable step sizes and to semigroups are given in Section 4. Two applications of our setting are given in Section 5. There we treat the Schrödinger equation, once with an external magnetic field and once with the harmonic potential.

\section{ANALYTIC FRAMEWORK AND CONVERGENCE}

Throughout the paper, $X$ will denote an arbitrary (complex) Banach space with norm $\|\cdot\|$. The corresponding operator norm will also be referred to as $\|\cdot\|$. Furthermore, $p$ denotes the classical order of this splitting method, $\mathcal{D}(F)$ denotes the domain of an operator $F$ in $X$, and $C$ will be a generic constant which assumes different values at different occurrences. Our analysis is built on the following two assumptions.

Assumption 2.1. The linear (possibly unbounded) operators $L, A$ and $B$, all generate $C_{0}$ groups on $X$, and the operators $A$ and $B$ satisfy in addition the bounds

$$
\left\|\mathrm{e}^{t A}\right\| \leq \mathrm{e}^{\omega|t|} \quad \text { and } \quad\left\|\mathrm{e}^{t B}\right\| \leq \mathrm{e}^{\omega|t|}
$$

for some $\omega \geq 0$ and all $t \in \mathbb{R}$.

We refer to 14 for an introduction to the theory of $C_{0}$ groups. For splitting methods with positive coefficients, the above assumption can be weakened to include parabolic problems. This extension will be discussed in Section 4 below.

In our analysis, we will frequently encounter compositions of the operators $A$ and $B$ that consist of exactly $k$ factors. We will denote such terms generically by $E_{k}$. For instance,

$$
E_{3} \in\{A A A, A A B, A B A, B A A, A B B, B A B, B B A, B B B\} .
$$

Next, we state our regularity assumption on the exact solution.

Assumption 2.2. All expressions of the form $E_{p+1} u(t)$ are uniformly bounded on the interval $-T \leq t \leq T$ for some $T>0$.

In our examples below, we will verify this assumption by identifying an appropriate subspace $\mathcal{U} \subseteq \mathcal{D}\left(L^{p+1}\right)$ of initial values such that $E_{p+1} \mathrm{e}^{t L}: \mathcal{U} \rightarrow X$ is well defined and $E_{p+1} \mathrm{e}^{t L} u_{0}$ is uniformly bounded in $t \in[-T, T]$ for any $u_{0} \in \mathcal{U}$.

We are now in the position to state the main result of our paper. The theorem below states that, under an appropriate regularity assumption, any exponential 
splitting method of classical order $p$ will retain its order in the present framework of unbounded operators.

Theorem 2.3. For the numerical solution of (1.1), consider an exponential splitting method (1.2) of classical order $p$. If Assumptions 2.1 and 2.2 are valid, then

$$
\left\|\left(S^{n}-\mathrm{e}^{n h L}\right) u_{0}\right\| \leq C|h|^{p}, \quad|n h| \leq T
$$

where the constant $C$ can be chosen uniformly on bounded time intervals and, in particular, independent of $n$ and $h$.

Proof. By applying the telescopic identity we obtain

$$
\left(S^{n}-\mathrm{e}^{n h L}\right) u_{0}=\sum_{\nu=0}^{n-1} S^{n-\nu-1}\left(S-\mathrm{e}^{h L}\right) \mathrm{e}^{\nu h L} u_{0} .
$$

Assumption 2.1 yields at once the stability bound $\|S\| \leq \mathrm{e}^{c \omega|h|}$, with a constant $c$ that only depends on the coefficients of the method. If we assume, for the time being, that the consistency bound

$$
\left\|\left(S-\mathrm{e}^{h L}\right) \mathrm{e}^{\nu h L} u_{0}\right\| \leq C|h|^{p+1}
$$

is valid, then the sought after error bound follows from

$$
\left\|\left(S^{n}-\mathrm{e}^{n h L}\right) u_{0}\right\| \leq \mathrm{e}^{c \omega T} \sum_{\nu=0}^{n-1}\left\|\left(S-\mathrm{e}^{h L}\right) \mathrm{e}^{\nu h L} u_{0}\right\| \leq C|h|^{p} .
$$

The desired consistency bound (2.1) will be derived in the next section.

\section{Consistency Proof}

One of the main difficulties when proving convergence of splitting schemes in the context of unbounded operators is to establish a suitable consistency. We will present here a general methodology to derive consistency bounds of the form (2.1) for splittings of arbitrary order.

We start by introducing some useful notation and terminology. For a linear operator $F: \mathcal{D}(F) \subseteq X \rightarrow X$ generating a $C_{0}$ group and a scalar $h \in \mathbb{R}$, we define the (possibly unbounded) operator $f=h F$, and the bounded operators $\varphi_{0}=\mathrm{e}^{f}$ and

$$
\varphi_{k}=\int_{0}^{1} \mathrm{e}^{(1-\tau) f} \frac{\tau^{k-1}}{(k-1) !} \mathrm{d} \tau, \quad \text { for } k \geq 1
$$

Henceforth, we will always denote these bounded operators by the corresponding greek letters. From this definition it is straightforward to prove the recurrence relation

$$
\varphi_{k}=\frac{1}{k !} I+f \varphi_{k+1}, \quad k \geq 0 .
$$

This relation will be a key tool for our analysis. An immediate implication is that the operator $f \varphi_{k}: X \rightarrow X$ is bounded for all $k \geq 1$, and as $F$ is densely defined on $X$ one also obtains that the commutation

$$
f \varphi_{k}=\varphi_{k} f
$$

is well defined on the whole space $X$. Hence, $\varphi_{k} f: X \rightarrow X$, with $k \geq 1$, is a bounded operator as well. 
With the syntax at hand, we define

$$
a=\gamma h A, \quad b=\delta h B, \quad \text { and } \quad \ell=h L,
$$

for $\gamma, \delta \in \mathbb{R}$, and the corresponding operators $\alpha_{k}, \beta_{k}, \lambda_{k}$. When required, we also use the more precise notation

$$
a^{[j]}=\gamma_{j} h A \quad \text { and } \quad b^{[j]}=\delta_{j} h B .
$$

The related operators are then denoted by $\alpha_{k}^{[j]}$ and $\beta_{k}^{[j]}$. Via the recurrence relation (3.1), we derive the following algebraic relations which we collect for later use.

Lemma 3.1. For $k \geq 1$, the following assertions hold:

(i) $\alpha_{k}=\frac{1}{k !} \alpha_{0}+\left(\alpha_{k+1}-\frac{1}{k !} \alpha_{1}\right) a$.

(ii) $\beta_{k}=\frac{1}{k !} \beta_{0}+\left(\beta_{k+1}-\frac{1}{k !} \beta_{1}\right) b$.

Proof. This follows at once from (3.1) by expressing the identity from the corresponding relation for $k=0$, i.e. $I=\alpha_{0}-\alpha_{1} a$ in the first assertion.

Lemma 3.2. For $m \geq 0$ and $k \geq 1$, the following assertions hold:

(i) $\alpha_{k} a \beta_{m}=\alpha_{k} \beta_{m} a+\alpha_{k} a \beta_{m+1} b-\alpha_{k} \beta_{m+1} b a$ on $\mathcal{D}(A)$.

(ii) $\beta_{k} b \alpha_{m}=\beta_{k} \alpha_{m} b+\beta_{k} b \alpha_{m+1} a-\beta_{k} \alpha_{m+1} a b \quad$ on $\mathcal{D}(B)$.

Proof. The first assertion follows from (3.1) and the identities

$$
\alpha_{k} a\left(\beta_{m}-\beta_{m+1} b\right)=\frac{1}{m !} \alpha_{k} a=\alpha_{k}\left(\beta_{m}-\beta_{m+1} b\right) a
$$

on $\mathcal{D}(A)$, and the second one is proven in the same way.

Lemma 3.3. For any $q \geq 0$, the following identity holds on $\mathcal{D}\left(L^{q+1}\right)$ :

$$
I-\sum_{m=0}^{q} \frac{(-1)^{m}}{m !} h^{m} L^{m} \lambda_{0}=h^{q+1} \sum_{m=0}^{q} \frac{(-1)^{m+1}}{m !} \lambda_{q-m+1} L^{q+1} .
$$

Proof. This identity follows by a simple induction argument. For $q=0$, the assertion reduces to $I-\lambda_{0}=-\lambda_{1} \ell$, which is again (3.1) for $k=0$.

In the induction step, we obtain

$$
I-\sum_{m=0}^{q+1} \frac{(-1)^{m}}{m !} h^{m} L^{m} \lambda_{0}=h^{q+1} \sum_{m=0}^{q+1} \frac{(-1)^{m+1}}{m !} \lambda_{q-m+1} L^{q+1} .
$$

Using once more (3.1), namely in the form

$$
\lambda_{q-m+1}=\frac{1}{(q-m+1) !} I+\lambda_{q-m+2} h L,
$$

gives the desired result.

We are now in the position to prove the consistency bound (2.1).

Theorem 3.4. Under Assumptions 2.1 and 2.2, we have the consistency bound

$$
\left\|\left(S-\mathrm{e}^{h L}\right) \mathrm{e}^{\nu h L} u_{0}\right\| \leq C|h|^{p+1}
$$

with a constant $C$ that can be chosen uniformly on bounded time intervals and, in particular, independent of $\nu$ and $h$. 
Proof. First, replace all factors of the time stepping operator $S$ for which the parameters $\gamma_{j}$ or $\delta_{j}$ are equal to zero by identities. If the number of stages $s$ is rescaled, we obtain one of the following three principle types of time stepping operators:

$$
S=\left\{\begin{array}{c}
\alpha_{0}^{[1]} \beta_{0}^{[1]} \ldots \alpha_{0}^{[s]} \beta_{0}^{[s]}, \\
\beta_{0}^{[1]} \ldots \alpha_{0}^{[s]} \beta_{0}^{[s]} \\
\alpha_{0}^{[1]} \beta_{0}^{[1]} \ldots \alpha_{0}^{[s]} .
\end{array}\right.
$$

Without any loss of generality, we will assume that $S$ is of the first type.

As a technical tool, we will need linear combinations of operators of the form

$$
T_{m, q}=\alpha^{[1]} \hat{a}^{[1]} \beta^{[1]} \hat{b}^{[1]} \ldots \alpha^{[s]} \hat{a}^{[s]} \beta^{[s]} \hat{b}^{[s]} E_{q},
$$

with $\hat{a}^{[k]}=a^{[k]}$ or $\hat{a}^{[k]}=I$, and $\hat{b}^{[k]}=b^{[k]}$ or $\hat{b}^{[k]}=I$. The first index $m$ of $T_{m, q}$ is the number of operators $\hat{a}^{[k]}$ and $\hat{b}^{[k]}$ which are not equal to $I$. Again, we have used a generic notation that captures the essential features of these operators. As a minor technical convention, operators of the form $T_{m, q}$ with $\hat{b}^{[s]}=b^{[s]}$ are by definition interpreted as being of the form $T_{m-1, q+1}$ when $0 \leq q \leq p$. The order of an operator of the form $T_{m, q}$ is defined as

$$
\operatorname{ord} T_{m, q}=m+q,
$$

and any linear combination of operators of the form $T_{m, q}$ will be denoted by $\mathcal{T}_{m, q}$.

The main step of our proof will be to write

$$
\mathrm{e}^{h L}=\left(S Q+h^{p+1} R\right) \mathrm{e}^{h L}
$$

with appropriate operators $Q$ and $R$. For this purpose, we consider the representation

$$
I=\prod_{j=1}^{s}\left(\alpha_{1}^{[j]}-\alpha_{2}^{[j]} a^{[j]}\right)\left(\beta_{1}^{[j]}-\beta_{2}^{[j]} b^{[j]}\right)
$$

which follows again from (3.1). Writing out this product yields a linear combination of operators of the form $T_{m, 0}$ and $T_{m, 1}$. The idea is now to transform these operators into terms of the form $h^{r} S E_{r}$, with $0 \leq r \leq p$, or into remainder terms of the form $h^{p+1} T_{k, p+1}$, with $0 \leq k \leq 2 s-1$. This is achieved by iteratively applying the following two reduction steps.

Step 1. For every term of the form $T_{0, r}$ with $0 \leq r \leq p$, apply Lemma 3.1 from the left to the first operator $\alpha_{k}$ or $\beta_{k}$, with $k>0$. This procedure replaces the old term with a new $T_{0, r}$ term and two additional terms of the form $T_{1, r}$ or $T_{0, r+1}$. Applying this procedure, at most $2 s$ times, yields one $S E_{r}$ term and higher order terms of the form $T_{1, r}$ or $T_{0, r+1}$.

Step 2. For every term of the form $T_{m, q}$, with $m \geq 1$ and $q \leq p$, apply Lemma 3.2 to the first sequence $\alpha a \beta$ or $\beta b \alpha$ found from the right. Denote the index of the encountered $a$ or $b$ by $\mu$. This replacement procedure generates three terms. One of them is either of the form $T_{m-1, q+1}$ or $T_{m, q}$, where terms of the latter form have the operator $a$ or $b$ shifted to a position with index greater than $\mu$, i.e., to the right. The remaining two terms are either of the form $T_{m, q+1}$ or $T_{m+1, q}$, both of higher order with a new operator $a$ or $b$ created in a position with index greater than $\mu$. Repeating this procedure a finite number of times eventually reduces the operator $T_{m, q}$ to a linear combination of operators of the form $T_{0, r}$ with $q+1 \leq r \leq p$ and remainder terms of the form $T_{k, p+1}$ with $0 \leq k \leq 2 s-1$. 
As we are increasing the order in all steps, the whole procedure will, after a finite number of iterations, produce the following representation

$$
I=\sum_{q=0}^{p} h^{q} S \mathcal{E}_{q}+h^{p+1} \sum_{k=0}^{2 s-1} \mathcal{T}_{k, p+1},
$$

where $\mathcal{E}_{q}$ denotes a certain linear combination of operators of the type $E_{q}$. Multiplying this relation from the right with $\mathrm{e}^{h L}$ gives

$$
S-\mathrm{e}^{h L}=S\left(I-\sum_{q=0}^{p} h^{q} \mathcal{E}_{q} \mathrm{e}^{h L}\right)-h^{p+1} \sum_{k=0}^{2 s-1} \mathcal{T}_{k, p+1} \mathrm{e}^{h L} .
$$

As all resulting operators of the form $\alpha^{[1]} \hat{a}^{[1]} \beta^{[1]} \hat{b}^{[1]} \ldots \alpha^{[s]} \hat{a}^{[s]} \beta^{[s]} \hat{b}^{[s]}$ are bounded on $X$, the consistency bound follows from Assumption 2.2, whenever

$$
I-\sum_{q=0}^{p} h^{q} \mathcal{E}_{q} \mathrm{e}^{h L}=h^{p+1} P \mathcal{E}_{p+1} \mathrm{e}^{h L}
$$

for some bounded operator $P: X \rightarrow X$. If we merely consider the current proof for the nonstiff case (let for example $L, A$ and $B$ be matrices of a fixed size), it follows by (3.3) that

$$
e^{-h L}-\sum_{q=0}^{p} h^{q} \mathcal{E}_{q}=\left(I-\sum_{q=0}^{p} h^{q} \mathcal{E}_{q} \mathrm{e}^{h L}\right) \mathrm{e}^{-h L}=\mathcal{O}\left(h^{p+1}\right),
$$

as the splitting method is of classical order $p$. Recall that terms $\mathcal{E}_{q}$ are polynomials in $A$ and $B$ of degree $q$. Thus, by a Taylor expansion of $\mathrm{e}^{-h L}$, one has the identification

$$
\mathcal{E}_{q}=\frac{(-1)^{q}}{q !} L^{q}
$$

for the terms in (3.2). As this equality is a pure algebraic relation, it is also valid for the general case with unbounded operators. The desired relation (3.4) now follows via Lemma 3.3

Remark 3.5. It seems to be more natural (and also simpler) to start the above proof with the identity

$$
I=\prod_{j=1}^{s}\left(\alpha_{0}^{[j]}-\alpha_{1}^{[j]} a^{[j]}\right)\left(\beta_{0}^{[j]}-\beta_{1}^{[j]} b^{[j]}\right) .
$$

Indeed, this is possible for $s=1$ and for the Strang splitting. In general, however, the arising term containing

$$
\alpha_{1} a \beta_{0} \alpha_{0} \beta_{0}
$$

cannot be reduced, as $\alpha_{1} \beta_{0} a \alpha_{0} \beta_{0}$ is unbounded; see Lemma 3.2

We close this section with an example that illustrates the ideas of the above proof for a concrete situation. We choose $s=1$ and $\gamma_{1}=\delta_{1}=1$ which corresponds to the first order exponential Lie splitting

$$
S=\mathrm{e}^{h A} \mathrm{e}^{h B}=\alpha_{0} \beta_{0} .
$$

Expanding the product $I=\left(\alpha_{1}-\alpha_{2} a\right)\left(\beta_{1}-\beta_{2} b\right)$ gives the terms

$$
\alpha_{1} \beta_{1}-\alpha_{2} a \beta_{1}-\alpha_{1} \beta_{2} b+\alpha_{2} a \beta_{2} b
$$


of the forms $T_{0,0}, T_{1,0}, T_{0,1}$, and $T_{1,1}$, respectively. Note that in this particular case, one can derive a shorter proof by using the expansion presented in Remark 3.5. Even so, we omit this as it does not illuminate our proof technique.

We start by applying the reduction step 1 to the terms of the form $T_{0, r}$, i.e., $\alpha_{1} \beta_{1}$ and $-\alpha_{1} \beta_{2} b$. First,

$$
\alpha_{1} \beta_{1}=\alpha_{0} \beta_{1}+\alpha_{2} a \beta_{1}-\alpha_{1} a \beta_{1},
$$

which results in a new $T_{0,0}$ term and two new $T_{1,0}$ terms. Applying this reduction once more to the newly created $T_{0,0}$ term gives a desired term $S E_{0}$ and two new $T_{0,1}$ terms, as

$$
\alpha_{0} \beta_{1}=\alpha_{0} \beta_{0}+\alpha_{0} \beta_{2} b-\alpha_{0} \beta_{1} b .
$$

Summing up gives us the representation

$$
\alpha_{1} \beta_{1}=\alpha_{0} \beta_{0}+\alpha_{0} \beta_{2} b-\alpha_{0} \beta_{1} b+\alpha_{2} a \beta_{1}-\alpha_{1} a \beta_{1} .
$$

Applying reduction step 1 twice to the term $-\alpha_{1} \beta_{2} b$ yields

$$
-\alpha_{1} \beta_{2} b=-\frac{1}{2} \alpha_{0} \beta_{0} b-\alpha_{2} a \beta_{2} b+\alpha_{1} a \beta_{2} b-\alpha_{0} \beta_{3} b^{2}+\frac{1}{2} \alpha_{0} \beta_{1} b^{2},
$$

and we conclude the reduction step 1 by collecting all the terms:

$I=\alpha_{0} \beta_{0}-\frac{1}{2} \alpha_{0} \beta_{0} b+\left(\alpha_{0} \beta_{2} b-\alpha_{0} \beta_{1} b\right)-\alpha_{1} a \beta_{1}+\alpha_{1} a \beta_{2} b+\left(-\alpha_{0} \beta_{3} b^{2}+\frac{1}{2} \alpha_{0} \beta_{1} b^{2}\right)$.

In order to proceed with reduction step 2 , we need to consider terms of the form $T_{m, q}$ with $m \geq 1$ and $q \leq 1$, i.e., $-\alpha_{1} a \beta_{1}$ and $\alpha_{1} a \beta_{2} b$. Applying Lemma 3.2 to the first term gives us

$$
-\alpha_{1} a \beta_{1}=-\alpha_{1} \beta_{1} a-\alpha_{1} a \beta_{2} b+\alpha_{1} \beta_{2} b a,
$$

and we have thereby developed a $T_{1,0}$ term into three terms of the forms $T_{0,1}, T_{1,1}$, and $T_{0,2}$, respectively. By coincidence, the $T_{1,1}$ term in the expansion of $-\alpha_{1} a \beta_{1}$ cancels out the term $\alpha_{1} a \beta_{2} b$, and step 2 is therefore completed. Hence, this first iteration results in the representation

$$
\begin{aligned}
I & =\alpha_{0} \beta_{0}-\frac{1}{2} \alpha_{0} \beta_{0} b+\left(\alpha_{0} \beta_{2} b-\alpha_{0} \beta_{1} b-\alpha_{1} \beta_{1} a\right)+\left(-\alpha_{0} \beta_{3} b^{2}+\frac{1}{2} \alpha_{0} \beta_{1} b^{2}+\alpha_{1} \beta_{2} b a\right) \\
& =S \mathcal{E}_{0}+h S \mathcal{E}_{1}+h \mathcal{T}_{0,1}+h^{2} \mathcal{T}_{0,2},
\end{aligned}
$$

and we have eliminated all $T_{0,0}$ terms. In the next (and final) iteration, we apply reduction step 1 to the three terms of the form $T_{0,1}$, and thereafter perform another step 2. This finally results in the sought after representation

$$
I=S(I-h L)+h^{2}\left(\mathcal{I}_{0,2}+\mathcal{T}_{1,2}\right)
$$

\section{Generalizations}

The aim of this section is to collect some extensions to the above results. In particular, we illustrate once more the general applicability of our approach. 
4.1. More operators. The above approach is not limited to splitting up into two operators. It can easily be generalized to splitting methods of the form

$$
S=\prod_{j=1}^{s} \prod_{m=1}^{M} \mathrm{e}^{\gamma_{j, m} h A_{m}}
$$

where $L=A_{1}+\cdots+A_{M}$. The only modification required in order to extend the consistency proof concerns Lemma 3.2. Its proof relies on representing the identity operator by relation (3.1), which now has to be generalized. In the case of three operators $L=A+B+F$, we make use of the representation

$$
\begin{aligned}
\frac{1}{m ! q !} I & =\left(\beta_{m}-\beta_{m+1} b\right)\left(\varphi_{q}-\varphi_{q+1} f\right) \\
& =\beta_{m} \varphi_{q}-\beta_{m+1} b \varphi_{q}-\beta_{m} \varphi_{q+1} f+\beta_{m+1} b \varphi_{q+1} f .
\end{aligned}
$$

The related commutator relation is then

$$
\begin{aligned}
\alpha_{k} a \beta_{m} \varphi_{q}=\alpha_{k} \beta_{m} \varphi_{q} a & +\alpha_{k} a \beta_{m+1} b \varphi_{q}+\alpha_{k} a \beta_{m} \varphi_{q+1} f-\alpha_{k} a \beta_{m+1} b \varphi_{q+1} f \\
& -\alpha_{k} \beta_{m+1} b \varphi_{q} a-\alpha_{k} \beta_{m} \varphi_{q+1} f a+\alpha_{k} \beta_{m+1} b \varphi_{q+1} f a,
\end{aligned}
$$

which is valid on $\mathcal{D}(A)$. This, together with the two other commutator relations, enables us once again to shift operators to the right. The consistency proof therefore follows along the same lines as in the case of two operators, and the generalization to more than three operators is now obvious.

4.2. More general splittings. The consistency of linear combinations of exponential splittings can be studied with our techniques as well. As an example, consider the fourth order splitting

$$
S=\frac{4}{3} S_{\frac{h}{2}} S_{\frac{h}{2}}-\frac{1}{3} S_{h}
$$

which is obtained by extrapolating the Strang splitting $S_{h}=\mathrm{e}^{\frac{h}{2} A} \mathrm{e}^{h B} \mathrm{e}^{\frac{h}{2} A}$. Our consistency proof applies to each term of (4.1) separately. We note that stability might be a serious problem for such types of splittings. A stability result for (4.1), however, is given in $[5]$.

4.3. Variable step sizes. Furthermore, our approach extends at once to variable step sizes. Consider again the case of two operators, for which the variable step size formulation is built on the operators

$$
S_{\nu}=\prod_{j=1}^{s} \mathrm{e}^{\gamma_{j} h_{\nu} A} \mathrm{e}^{\delta_{j} h_{\nu} B} .
$$

The following corollary follows from the observation that the step size $h$ only plays the role of a parameter in the proof of the consistency.

Corollary 4.1. For the numerical solution of (1.1), consider an exponential splitting method (4.2) of classical order $p$. If Assumptions 2.1 and 2.2 are valid, then

$$
\left\|\left(S_{n} \ldots S_{1}-\mathrm{e}^{\left(h_{1}+\cdots+h_{n}\right) L}\right) u_{0}\right\| \leq C \sum_{\nu=1}^{n}\left|h_{\nu}\right|^{p+1}, \quad\left|h_{1}+\cdots+h_{n}\right| \leq T,
$$

where the constant $C$ can be chosen uniformly on bounded time intervals and, in particular, independent of the chosen step size sequence. 
4.4. Semigroups. If all coefficients $\gamma_{j}$ and $\delta_{j}$ are positive, then the splitting operator $S$ can even be defined for generators of $C_{0}$ semigroups. They arise, for example, in the context of parabolic problems. We refer to [14 for an introduction to the theory of $C_{0}$ semigroups. In this setting, Theorem 2.3 can be rephrased on bounded time intervals $0 \leq n h \leq T$ by replacing Assumption 2.2 by the following one.

Assumption 4.2. The linear operators $L, A$ and $B$, all generate $C_{0}$ semigroups on $X$, and the operators $A$ and $B$ satisfy, in addition, the bounds

$$
\left\|\mathrm{e}^{t A}\right\| \leq \mathrm{e}^{\omega t} \text { and }\left\|\mathrm{e}^{t B}\right\| \leq \mathrm{e}^{\omega t}
$$

for some $\omega \in \mathbb{R}$ and all $t \geq 0$.

We finally note that the positivity requirement on the coefficients restricts the splitting method to be of second order at most; see [1. A prominent example of a second order method with positive coefficients is the Strang splitting where $\gamma_{1}=\gamma_{2}=1 / 2, \delta_{1}=1$ and $\delta_{2}=0$.

\section{EXAMPLES}

In order to exemplify how the derived convergence results may be used in practice, we will devote this section to the validation of Assumptions 2.1 and 2.2 in the context of the Schrödinger equation with different types of potentials. A brief background to the presented examples can be found in [3, Chapter I.A, Sections 4.3 and 6.2$]$ and [17.

5.1. The Schrödinger equation with an external magnetic field. The wave function $u$ of a single negatively charged particle in $\mathbb{R}^{3}$ exposed to a magnetic field $b(x)=\nabla \times a(x)$ and a potential $W(x)=V(x)-|a(x)|^{2}$ can be modeled as

$$
\mathrm{i} \dot{u}=(-\mathrm{i} \nabla-a(x))^{2} u+W(x) u .
$$

Note that physical parameters, such as mass and electric charge, have been omitted in the equation as these (real) quantities do not alter the analysis. If the vector potential $a(x)$ is assumed to satisfy the Coulomb condition, i.e., $\nabla \cdot a(x)=0$, we can rewrite the above equation as $\dot{u}=L u=(A+B) u$, with

$$
A=\mathrm{i} \Delta \quad \text { and } \quad B=2 a(x) \cdot \nabla-\mathrm{i} V(x) .
$$

As the analysis itself is not restricted to $\mathbb{R}^{3}$, we consider the problem on $\mathbb{R}^{d}, d \geq 1$, and assume that $a: \mathbb{R}^{d} \rightarrow \mathbb{R}^{d}$ and $V: \mathbb{R}^{d} \rightarrow \mathbb{R}$ are real $2 p$ times continuously differentiable functions with bounded derivatives. The aim is now to validate Assumptions 2.1 and 2.2 for $X=L^{2}\left(\mathbb{R}^{d}\right)$.

The standard approach to prove that the operators $L, A$ and $B$ all generate unitary groups is to first prove that the imaginary unit i times the highest order differential operators, i.e,

$$
-\Delta \text { and } 2 \mathrm{i} a(x) \cdot \nabla,
$$

are selfadjoint on $X$. Second, observe that the operators (5.1) remain selfadjoint (with the same domains) when perturbed by symmetric lower order differential operators. We refer to [3, Proposition IX.B.2.2 and Example IX.B.2.2d] for further details. The desired generating properties of $L, A$ and $B$ then follow by Stone's theorem [14, Theorem 1.10.8].

To prove the selfadjointness of the operators (5.1), it is convenient to recall that a densely defined and symmetric operator $F$ on a Hilbert space is selfadjoint 
whenever the range condition $\mathcal{R}(F \pm \mathrm{i} I)=X$ is fulfilled [15, Theorem VIII.3]. The operators $-\Delta: H^{2}\left(\mathbb{R}^{d}\right) \rightarrow X$ and $2 \mathrm{i} a(x) \cdot \nabla: H^{1}\left(\mathbb{R}^{d}\right) \rightarrow X$ are both densely defined and symmetric. The latter follows simply by integrating by parts. A Fourier transformation yields that $\mathcal{R}(-\Delta \pm \mathrm{i} I)=X$, and $-\Delta: H^{2}\left(\mathbb{R}^{d}\right) \rightarrow X$ is therefore a selfadjoint operator. Finally, we note that the closure of $2 \mathrm{i} a(x) \cdot \nabla: H^{1}\left(\mathbb{R}^{d}\right) \rightarrow X$ is again symmetric and it satisfies the range condition, which is a consequence of the general theory of symmetric hyperbolic systems [6, Theorem 3.5.2]. Hence, $2 \mathrm{i} a(x) \cdot \nabla$ is selfadjoint when equipped with a (possibly) larger domain than $H^{1}\left(\mathbb{R}^{d}\right)$.

With the above results, we obtain that Assumption 2.1 is valid. Next, consider Assumption 2.2 and choose $\mathcal{U}=H^{2(p+1)}\left(\mathbb{R}^{d}\right)=\mathcal{D}\left(L^{p+1}\right)$. As $\mathrm{e}^{t L}: \mathcal{D}\left(L^{p+1}\right) \rightarrow$ $\mathcal{D}\left(L^{p+1}\right)$ and the operator $E_{p+1}$ is merely a differential operator of (at most) order $2(p+1)$, it also holds true that $E_{p+1} \mathrm{e}^{t L} u_{0}:[-T, T] \rightarrow X$ is uniformly bounded for all $u_{0} \in \mathcal{U}$, which validates the assumption.

5.2. The Schrödinger equation with a harmonic potential. The splitting results can also be applied to the Schrödinger equation with an unbounded potential. To this end, consider the equation related to the harmonic oscillator,

$$
\mathrm{i} \dot{u}=-\Delta u+|x|^{2} u,
$$

which typically arises when modeling the vibrations of molecules. We have again omitted the related physical parameters in the equation. Writing the equation in the form $\dot{u}=L u=(A+B) u$ gives us

$$
A=\mathrm{i} \Delta \quad \text { and } \quad B=-\mathrm{i}|x|^{2} .
$$

Let $X=L^{2}\left(\mathbb{R}^{d}\right)$. The operator $A: H^{2}\left(\mathbb{R}^{d}\right) \rightarrow X$ then generates a unitary group, as shown in the previous example, and the same trivially holds true for the operator $B:\left\{v \in X:|x|^{2} v \in X\right\} \rightarrow X$, as

$$
\left(\mathrm{e}^{t B} u_{0}\right)(x)=\mathrm{e}^{-\mathrm{i} t|x|^{2}} u_{0}(x) .
$$

Next, we define the ( $d$-dimensional) Hermite functions $\left\{H_{k}\right\}$ as the tensor products of their one-dimensional counterparts. The generating properties of the full operator $L:\{v \in X: L v \in X\} \rightarrow X$ then follow by observing that $\left\{H_{k}\right\}$ constitutes an eigenbasis to $L$ in $X$, and the related group can then be expressed as

$$
\mathrm{e}^{t L} u_{0}=\sum_{k} c_{k}\left[u_{0}\right] \mathrm{e}^{-\mathrm{i} t \lambda_{k}} H_{k}, \quad \text { where } \quad u_{0}=\sum_{k} c_{k}\left[u_{0}\right] H_{k}
$$

and $\lambda_{k}$ are the related eigenvalues. For complete proofs see [3, Section XV.5.2]. Note that the unitary property follows by Parseval's equality. This verifies Assumption 2.1.

To validate Assumption 2.2. we momentarily restrict our attention to the onedimensional case, $d=1$. Here, the Hermite functions are defined as

$$
H_{k}(x)=\frac{(-1)^{k}}{\sqrt{2^{k} k ! \sqrt{\pi}}} \mathrm{e}^{x^{2} / 2} D^{k} \mathrm{e}^{-x^{2}}, \quad \text { for } k \in \mathbb{N},
$$

where $D=\mathrm{d} / \mathrm{d} x$. These functions are known to satisfy the relations

$$
x H_{k}=\sqrt{k / 2} H_{k-1}+\sqrt{(k+1) / 2} H_{k+1}
$$

and

$$
D H_{k}=\sqrt{k / 2} H_{k-1}-\sqrt{(k+1) / 2} H_{k+1},
$$


for $k \geq 0$. Thus, terms of the form $A H_{k}$ and $B H_{k}$ can always be given a finite representation in the Hermite basis. This observation yields that $E_{p+1}$ may be characterized as

$$
E_{p+1} v=\lim _{K \rightarrow \infty} E_{p+1} \sum_{k=0}^{K} c_{k}[v] H_{k}=\sum_{k \in \mathbb{N}} c_{k}[v] E_{p+1} H_{k}=\sum_{k \in \mathbb{N}} \tilde{c}_{k}[v] H_{k},
$$

with new coefficients $\tilde{c}_{k}$, and the domain of the operator in $X$ is now readily

$$
\mathcal{D}\left(E_{p+1}\right)=\left\{v \in X: \sum_{k \in \mathbb{N}}\left|\tilde{c}_{k}[v]\right|^{2}<\infty\right\} .
$$

The representation (5.2) together with Parseval's equality implies that the group $\mathrm{e}^{t L}$ is invariant over $\mathcal{D}\left(E_{p+1}\right)$ for all $t \in[-T, T]$, and Assumption 2.2 is therefore valid in $\mathbb{R}$, with $\mathcal{U}=\mathcal{D}\left(E_{p+1}\right)$. The very same argumentation can be used in $\mathbb{R}^{d}$, $d>1$, as the higher dimensional Hermite functions are merely compositions of the one-dimensional ones.

\section{REFERENCES}

[1] S. Blanes and F. Casas, On the necessity of negative coefficients for operator splitting schemes of order higher than two, Appl. Numer. Math. 54 (2005), 23-37. MR2134093(2006b:65085)

[2] M. Crandall and A. Majda, The method of fractional steps for conservation laws, Numer. Math. 34 (1980), 285-314. MR571291 (81j:65101)

[3] R. Dautray and J.-L. Lions, Mathematical Analysis and Numerical Methods for Science and Technology. Vol. 1/3/5, Springer, Berlin, 1990/90/92. MR1036731 (90k:00004)

[4] S. Descombes and M. Schatzman, Strang's formula for holomorphic semi-groups, J. Math. Pures Appl. 81 (2002), 93-114. MR1994884 (2005g:35008)

[5] B. O. Dia and M. Schatzman, Commutateurs de certains semi-groupes holomorphes et applications aux directions alternées, M2AN Math. Model. Numer. Anal. 30 (1996), 343-383. MR:1391710 (97e:47055)

[6] H. O. Fattorini, The Cauchy Problem, Addison-Wesley, Reading, 1983. MR692768 (84g:34003)

[7] E. Hairer, Ch. Lubich, and G. Wanner, Geometric Numerical Integration. Structurepreserving Algorithms for Ordinary Differential Equations, second ed., Springer, Berlin, 2006. MR:2221614 (2006m:65006)

[8] E. Hansen and A. Ostermann, Dimension splitting for evolution equations, Numer. Math. 108 (2008), 557-570. MR2369204

[9] W. Hundsdorfer and J. Verwer, Stability and convergence of the Peaceman-Rachford ADI method for initial-boundary value problems, Math. Comp. 53 (1989), 81-101. MR969489 (90h:65195)

[10] T. Jahnke and Ch. Lubich, Error bounds for exponential operator splittings, BIT 40 (2000), 735-744. MR $1799313(2001 \mathrm{k}: 65143)$

[11] K. H. Karlsen and N. H. Risebro, An operator splitting method for nonlinear convectiondiffusion equations, Numer. Math. 77 (1997), 365-382. MR.1469677 (98i:65073)

[12] Ch. Lubich, On splitting methods for Schrödinger-Poisson and cubic nonlinear Schrödinger equations, Math. Comp. 77 (2008), 2141-2153. MR.2429878

[13] R. I. McLachlan and G. R. Quispel, Splitting methods, Acta Numer. 11 (2002), 341-434. MR2009376 (2004f:34001)

[14] A. Pazy, Semigroups of Linear Operators and Applications to Partial Differential Equations, Springer, New York, 1983. MR710486 (85g:47061)

[15] M. Reed and B. Simon, Methods of Modern Mathematical Physics. I. Functional Analysis, Academic Press, New York, 1972. MR0493419 (58:12429a)

[16] Z. H. Teng, On the accuracy of fractional step methods for conservation laws in two dimensions, SIAM J. Numer. Anal. 31 (1994), 43-63. MR.1259965 (95c:65141) 
[17] S. Teufel, Adiabatic Perturbation Theory in Quantum Dynamics, Lecture Notes in Mathematics, vol. 1821, Springer, Berlin, 2003. MR2158392 (2006g:81061)

[18] M. Thalhammer, High-order exponential operator splitting methods for time-dependent Schrödinger equations, SIAM J. Numer. Anal. 46 (2008), 2022-2038. MR2399406

Institut für Mathematik, Universität Innsbruck, Technikerstrasse 13, A-6020 InnsBRUCK, AUSTRIA

E-mail address: eskil.hansen@uibk.ac.at

Institut für Mathematik, Universität Innsbruck, Technikerstrasse 13, A-6020 InNsBRUCK, AUSTRIA

E-mail address: alexander.ostermann@uibk.ac.at 